\title{
Core/Double-Cone Emission-Beam Structure in a Millisecond Pulsar
}

\author{
Joanna M. Rankin \\ Physics Department, University of Vermont, Burlington, VT 05405 USA: \\ email: Joanna.Rankin@uvm.edu
}

\begin{abstract}
Slow pulsars show a great deal of qualitative and quantitative regularity in the structure of their radio emission beams as described by the core/double-cone model; however, millisecond pulsars (MSPs) have shown little. It is thus arresting to encounter a 2.7-s MSP with what appears to be a double-cone/core profile - and even more so to find that the arrangement of the cones around the core suggest aberration/retardation emission heights that are very reasonable. This and several other pulsars then represent rare opportunities for analysis and raise questions about why a few MSPs show such orderly beam structure while so many do not.
\end{abstract}

Keywords. (stars:) pulsars: individual (J0337+1715), radiation mechanisms: nonthermal

Millisecond pulsar (MSP) emission processes have remained enigmatic despite the prominence of MSPs as indispensable tools of contemporary physics and astrophysics. MSP magnetospheres are much more compact, and their magnetic fields are typically much weaker and probably more complex than those of normal pulsars, as a result of the period of accretion that is believed to recycle old pulsars into MSPs. Slow pulsars exhibit many regularities of profile form and polarization that together suggest an overall beam geometry comprised by two distinct emission cones and a central core beam (e.g., Rankin $1993 \mathrm{a}, \mathrm{b})$. These regularities apparently occur because the magnetic field is usually highly dipolar at the roughly 3-500-km height where radio emission occurs (Rankin, Melikidze \& Mitra 2018; in preparation).

The profile of millisecond pulsar J0337+1715 surprisingly has what appears to be a five-component core/double-cone configuration; see Figure 1. This five-component profile is important because it has not been clear whether MSPs ever exhibit such structure. The profile is also very asymmetric about the core component - and in just the manner that would be expected due to aberration/retardation. Note that the conal centers fall well before the core component. Further, the core appears to have enough width to reflect the full angular diameter of the pulsar's polar cap.

A full geometric and aberration/retardation (A/R) analysis of J0337+1715 and three other MSPs given in Rankin et al. (2017). J0337+1715's core component is found to have a width compatible with the $67^{\circ}$ expected if it reflects the full angular size of the pulsar's polar cap. Its cones are compatible with the structures of slower pulsars, and their A/R emission altitudes are measured to be some $70 \mathrm{~km}$ and $85 \mathrm{~km}$ within a velocity-of-light cylinder distance of $130 \mathrm{~km}$.

\section{References}

Rankin, J. M. 1993a, ApJ , 405, 285 (ET VIa)

Rankin, J. M. 1993b, ApJ Suppl., 85, 145 (ET VIb)

Rankin, J. M., Archibald, A., Hessels, J., van Leeuwen, J., Mitra, D., Ransom, S., Stairs, I., van Straten, W., \& Weisberg, J. M.2017, ApJ , 845, 23 

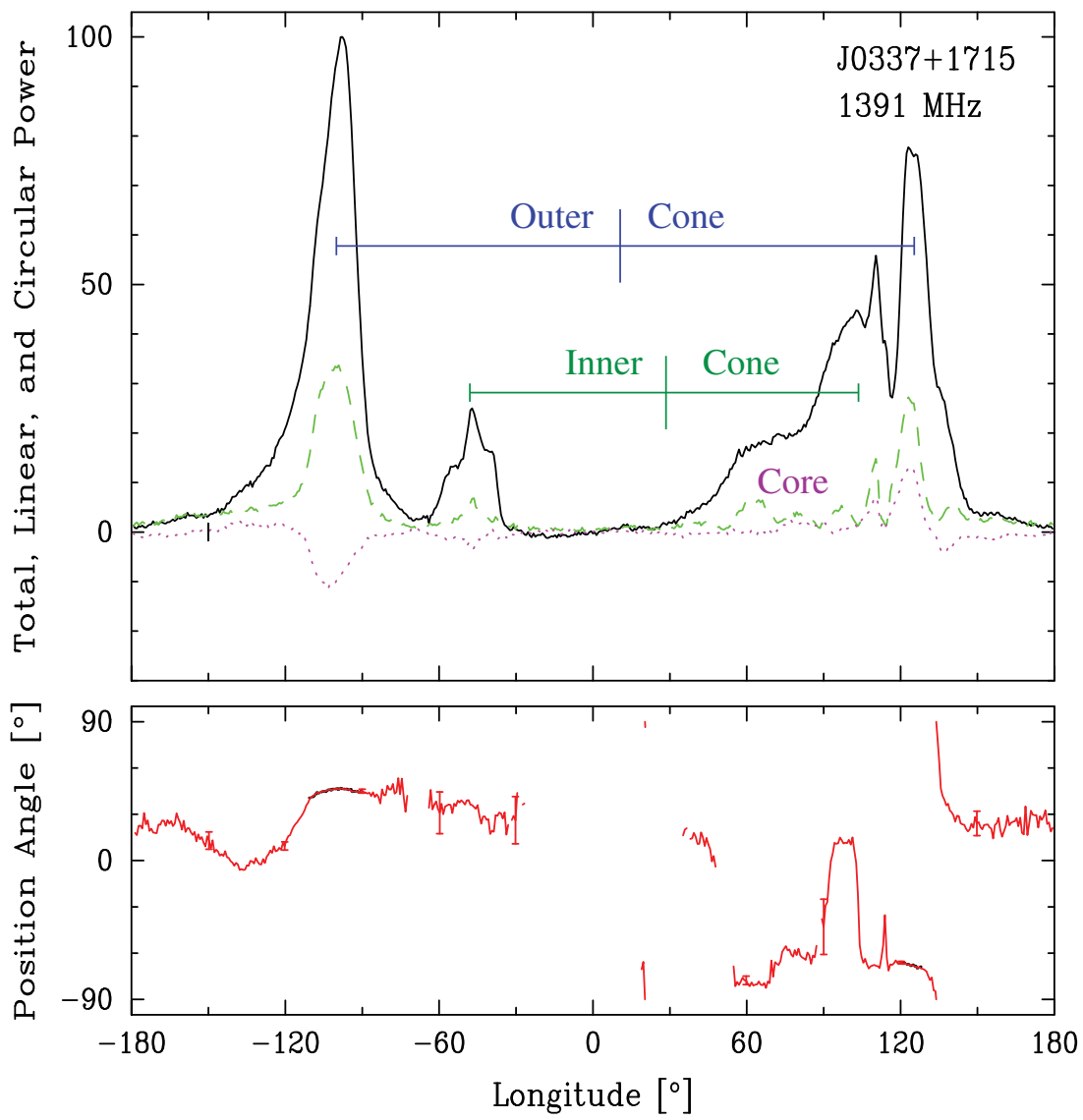

Figure 1. Total 1.4-GHz Arecibo PUPPI polarized profile of pulsar J0337+1715 showing its apparent core component as well as its pairs of inner and outer conal components. The core component seems to be conflated with the trailing conal components at about $90^{\circ}$ longitude. The positions and centers of the two conal component pairs are indicated. They are far from symmetrically spaced as in slower pulsars, apparently because of aberration/retardation. However, note that the outer cone center precedes the inner one as well as the core. The upper panel gives the total intensity (Stokes $I$; solid curve), the total linear $\left(L\left[=\sqrt{Q^{2}+U^{2}}\right]\right.$; dashed green), and the circular polarization (Stokes $V$; dotted magenta). The PPA $\left[=(1 / 2) \tan ^{-1}(U / Q)\right]$ values (lower panel) have been Faraday-derotated to infinite frequency. Errors in the PPAs were computed relative to the off-pulse noise phasor-that is, $\sigma_{P P A} \sim \tan ^{-1}\left(\sigma_{\text {off }}-\right.$ pulse $\left./ L\right)$ and are plotted when $<30^{\circ}$ and indicated by occasional 3- $\sigma$ error bars. 